\title{
Correction to: A suite of automated tools to quantify hand and wrist motor function after cervical spinal cord injury
}

\author{
Katelyn M. Grasse ${ }^{1,2}$, Seth A. Hays ${ }^{1,2,3^{*}}$, Kimiya C. Rahebi ${ }^{1}$, Victoria S. Warren ${ }^{1}$, Elizabeth A. Garcia ${ }^{1}$,
} Jane G. Wigginton', Michael P. Kilgard ${ }^{1,3}$ and Robert L. Rennaker $\|^{1,2,3}$

\section{Correction to: J NeuroEng Rehabil (2019) 16:48 https://doi.org/10.1186/s12984-019-0518-8}

The original article [1] contains several errors which the authors would like to rectify:

1) Figs $3 B$ \& $3 C$ contain duplicate data from Fig. 5 . The correct version of Fig. 3 can be viewed ahead.

2) The Authors' contributions section contains a minor typo and should instead read as the following: "KMG, SAH, JW, MPK and RLR contributed to experimental design. KMG and SAH wrote the manuscript. KMG and RLR provided engineering. KMG, KR, VW and EG conducted data collection. SAH, MPK, and RLR provided funding. All authors read and approved the final manuscript."

3) Tables $2 \& 3$ contain minor formatting errors. The correct presentation of both tables can be viewed ahead.

\section{Author details}

'The University of Texas at Dallas, Texas Biomedical Device Center800 West Campbell Road, Richardson, TX 75080-3021, USA. ${ }^{2}$ The University of Texas at Dallas, Erik Jonsson School of Engineering and Computer Science, 800 West Campbell Road, Richardson, TX 75080-3021, USA. ${ }^{3}$ The University of Texas at Dallas, School of Behavioral Brain Sciences, 800 West Campbell Road, Richardson, TX 75080-3021, USA.
Published online: 07 January 2020

\section{Reference}

1. Grasse KM, Hays SA, Rahebi KC, Warren VS, Garcia EA, Wiggington JG, et al. A suite of automated tools to quantify hand and wrist motor function after cervical spinal cord injury. J NeuroEng Rehabil. 2019;16:48. https://doi.org/ 10.1186/s12984-019-0518-8.

* Correspondence: seth.hays@utdallas.edu

The original article can be found online at https://doi.org/10.1186/s12984019-0518-8

'The University of Texas at Dallas, Texas Biomedical Device Center800 West Campbell Road, Richardson, TX 75080-3021, USA

${ }^{2}$ The University of Texas at Dallas, Erik Jonsson School of Engineering and Computer Science, 800 West Campbell Road, Richardson, TX 75080-3021, USA

Full list of author information is available at the end of the article

C The Author(s). 2019 Open Access This article is distributed under the terms of the Creative Commons Attribution 4.0 International License (http://creativecommons.org/licenses/by/4.0/), which permits unrestricted use, distribution, and reproduction in any medium, provided you give appropriate credit to the original author(s) and the source, provide a link to the Creative Commons license, and indicate if changes were made. The Creative Commons Public Domain Dedication waiver (http://creativecommons.org/publicdomain/zero/1.0/) applies to the data made available in this article, unless otherwise stated. 


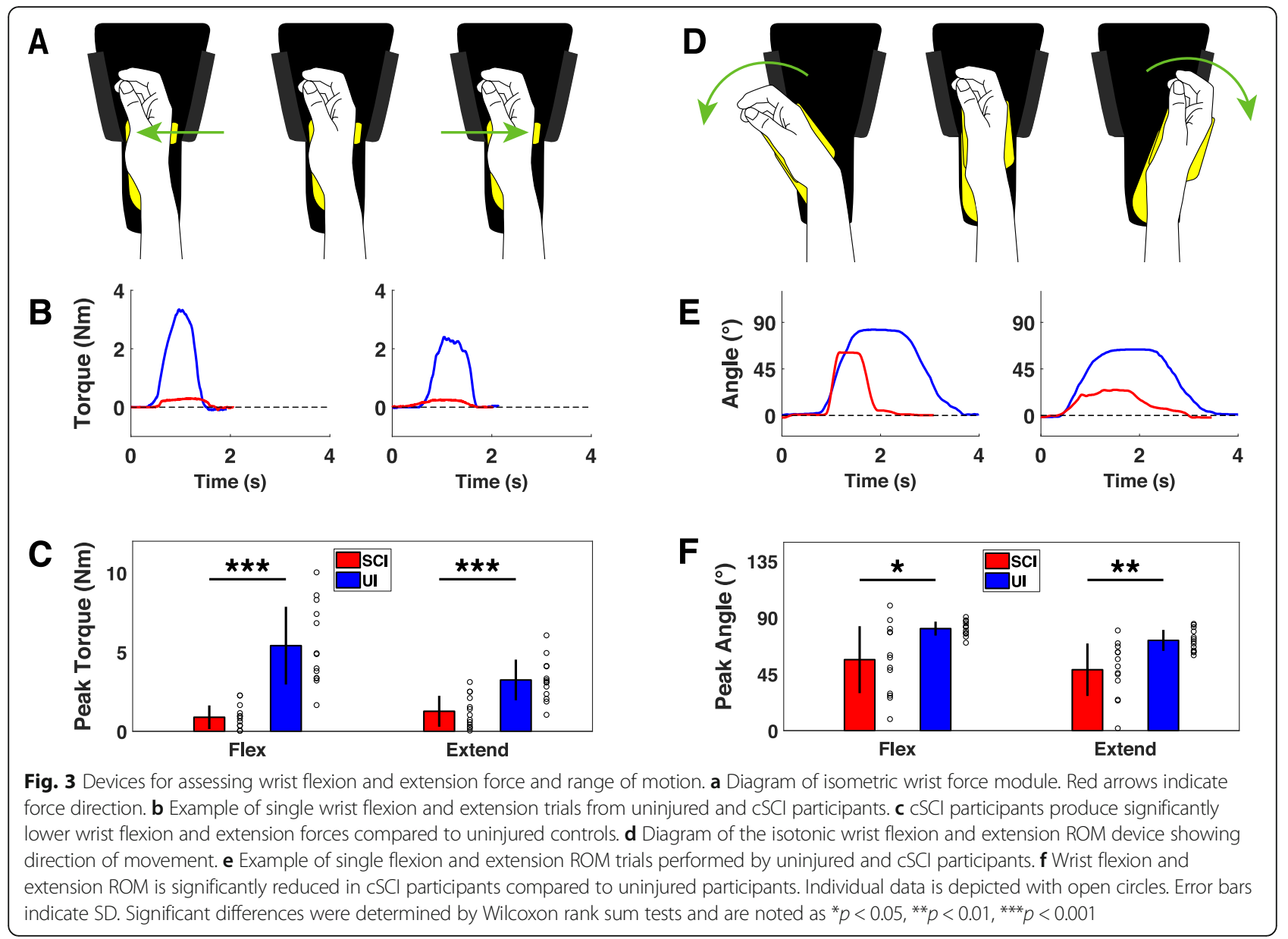

Table 2 Novel system measurement results by participant group $(N=13)$. CV, coefficient of variation; + Values based on $n=12$

\begin{tabular}{|c|c|c|c|c|c|c|c|}
\hline \multirow[b]{3}{*}{ Measure } & \multirow[b]{3}{*}{ Task (units) } & \multicolumn{3}{|l|}{ Peak } & \multicolumn{3}{|l|}{ CV } \\
\hline & & \multirow{2}{*}{$\begin{array}{l}\mathrm{UI} \\
\text { Mean (SD) }\end{array}$} & \multicolumn{2}{|l|}{$\mathrm{cSCl}$} & \multirow{2}{*}{$\begin{array}{l}\text { UI } \\
\text { Mean (SD) }\end{array}$} & \multicolumn{2}{|l|}{$\mathrm{CSCl}$} \\
\hline & & & Mean (SD) & $p$-val & & Mean (SD) & $p$-val \\
\hline \multirow[t]{8}{*}{ Force } & Finger Flexion $(\mathrm{N})^{\dagger}$ & $78.3(22.7)$ & $2.94(2.49)$ & $<0.001$ & $6.8(2.3)$ & $40.1(25.3)$ & $<0.001$ \\
\hline & Finger Extension $(\mathrm{N})^{\dagger}$ & $17.3(5.42)$ & $1.23(1.99)$ & $<0.001$ & $12.7(5.2)$ & $71.1(34.6)$ & $<0.001$ \\
\hline & Wrist Flexion (Nm) & $5.41(2.46)$ & $0.89(0.75)$ & $<0.001$ & $14.7(4.0)$ & $27.4(18.2)$ & 0.009 \\
\hline & Wrist Extension (Nm) & $3.24(1.29)$ & $1.27(0.98)$ & $<0.001$ & $12.4(4.6)$ & $25.7(12.8)$ & 0.002 \\
\hline & Handle Pronation (Nm) & $6.36(2.37)$ & $1.76(1.36)$ & $<0.001$ & $8.3(2.9)$ & $31.4(33.2)$ & 0.015 \\
\hline & Handle Supination (Nm) & $4.58(1.71)$ & $1.10(0.67)$ & $<0.001$ & $8.0(3.5)$ & $21.0(16.5)$ & 0.013 \\
\hline & Doorknob Pronation (Nm) & $3.63(1.14)$ & $0.30(0.28)$ & $<0.001$ & $10.3(3.6)$ & $60.7(44.9)$ & $<0.001$ \\
\hline & Doorknob Supination (Nm) & $3.51(1.40)$ & $0.38(0.32)$ & $<0.001$ & $11.6(5.9)$ & $28.6(13.3)$ & $<0.001$ \\
\hline \multirow[t]{6}{*}{ Range of Motion } & Wrist Flexion $\left(^{\circ}\right)$ & $81.3(5.56)$ & $56.5(26.7)$ & 0.027 & $4.0(2.7)$ & $8.4(7.8)$ & 0.11 \\
\hline & Wrist Extension $\left({ }^{\circ}\right)$ & $71.9(8.36)$ & $48.5(20.9)$ & 0.002 & $2.7(1.3)$ & $7.6(7.1)$ & 0.026 \\
\hline & Handle Pronation $\left({ }^{\circ}\right)$ & $104.1(12.6)$ & $95.9(36.9)$ & 0.92 & $4.5(2.4)$ & $6.5(4.4)$ & 0.87 \\
\hline & Handle Supination $\left(^{\circ}\right)$ & $74.0(14.5)$ & $56.8(24.2)$ & 0.051 & $5.3(1.6)$ & $4.3(2.4)$ & 0.31 \\
\hline & Doorknob Pronation $\left(^{\circ}\right)$ & $107.1(20.9)$ & $94.7(41.5)$ & 0.72 & $4.4(2.4)$ & $21.7(36.7)$ & 0.12 \\
\hline & Doorknob Supination $\left(^{\circ}\right)$ & $72.1(19.8)$ & $56.7(35.1)$ & 0.25 & $5.4(2.4)$ & $36.8(69.7)$ & 0.13 \\
\hline
\end{tabular}


Table 3 Test-retest reproducibility results of the novel metrics for $\mathrm{cSCl}$ participants $(N=10)$. MDD, minimally detectable difference; $\mathrm{SD}_{20}$, standard deviation of 20 trials; ICC, intraclass correlation coefficient; ${ }^{\dagger}$ Values based on $n=9$

\begin{tabular}{|c|c|c|c|c|c|c|}
\hline Measure & Task (units) & Change (SD) & $p$-val & MDD & $1.96 * \mathrm{SD}_{20}$ & ICC \\
\hline & Composite Score & $0.03(0.06)$ & 0.72 & 0.107 & - & 0.95 \\
\hline \multirow[t]{8}{*}{ Force } & Finger Flexion $(\mathrm{N})^{\dagger}$ & $0.21(3.42)$ & 0.88 & 5.99 & 2.52 & 0.40 \\
\hline & Finger Extension $(\mathrm{N})^{+}$ & $-0.08(1.99)$ & 0.94 & 3.48 & 1.29 & 0.63 \\
\hline & Wrist Flexion (Nm) & $0.65(1.99)$ & 0.45 & 3.70 & 0.96 & 0.45 \\
\hline & Wrist Extension (Nm) & $0.34(0.63)$ & 0.55 & 1.17 & 0.68 & 0.85 \\
\hline & Handle Pronation (Nm) & $0.13(1.37)$ & 0.88 & 2.55 & 1.10 & 0.75 \\
\hline & Handle Supination (Nm) & $0.16(0.16)$ & 0.75 & 1.79 & 0.76 & 0.62 \\
\hline & Doorknob Pronation (Nm) & $0.005(0.16)$ & 0.97 & 0.30 & 0.28 & 0.90 \\
\hline & Doorknob Supination (Nm) & $-0.05(0.22)$ & 0.65 & 0.41 & 0.24 & 0.67 \\
\hline \multirow[t]{6}{*}{ Range of Motion } & Wrist Flexion $\left(^{\circ}\right)$ & $1.52(10.8)$ & 0.90 & 20.1 & 10.8 & 0.93 \\
\hline & Wrist Extension $\left({ }^{\circ}\right)$ & $4.46(6.4)$ & 0.67 & 11.9 & 8.3 & 0.95 \\
\hline & Handle Pronation $\left({ }^{\circ}\right)$ & $3.49(18.7)$ & 0.84 & 34.7 & 15.5 & 0.88 \\
\hline & Handle Supination $\left({ }^{\circ}\right)$ & $0.07(7.6)$ & 0.99 & 14.0 & 7.4 & 0.96 \\
\hline & Doorknob Pronation $\left(^{\circ}\right)$ & $5.29(20.8)$ & 0.76 & 38.7 & 22.1 & 0.86 \\
\hline & Doorknob Supination $\left(^{\circ}\right)$ & $0.03(9.4)$ & 0.99 & 17.5 & 14.9 & 0.93 \\
\hline
\end{tabular}

